\title{
Out of Africa: Observations on the histopathology of Mycobacterium ulcerans infection
}

\author{
J Hayman
}

\section{Introduction}

Mycobacterium ulcerans infection produces progressive skin ulceration in man and other mammals. The disease was first described in 1948 in patients from the Bairnsdale district in Australia ${ }^{1}$ but was known in Africa well before this time. ${ }^{2}$ It has since been recorded in many, mostly tropical countries in Africa, Central and South America, and South East Asia. ${ }^{3}$ The infection characteristically occurs in defined areas which are associated with river or lacustrine systems draining tropical or warm-temperature rain forest. Like the flora to which it is related, the mycobacterium shows a Gondwanian distribution which is evidence of its great antiquity. ${ }^{4}$

The disease usually begins as a skin papule (stage 1A) which later ulcerates (stage 2) (fig $1)^{5}$; less commonly the infection may first appear as a subcutaneous nodule (stage 1B) ${ }^{6}$ which subsequently affects the dermis and then ulcerates, or sometimes the disease may produce diffuse oedema of a limb (stage 1C), ${ }^{7}$ due to a necrotising panniculitis, which clinically mimicks cellulitis. The infection is usually on an extremity but, particularly in children, it may affect the face or trunk. ${ }^{8}$ Occasionally, the necrotic process extends through deep fascia with involvement of muscle ${ }^{9}$ and even bone. ${ }^{10}$ Metastatic infection may occur.

The features of the pathology of $M$ ulcerans infection have been described ${ }^{1}$; additional characteristics of the disease, as observed in Zaire (Belgian Congo), have been detailed in the papers by Janssens et al. ${ }^{711}$ Infection in Uganda has been described by Dodge, ${ }^{12}{ }^{13}$ and later by Connor and Lunn, ${ }^{14}{ }_{15}$ and infection in Papua New Guinea by Lytton and Lavett. ${ }^{16}$ Hayman and McQueen described the pathology in additional Bairnsdale district patients in 1985. ${ }^{17}$ Burchard and Bierther ${ }^{18}$ briefly described the pathology of the disease as seen in Gabon; their paper was illustrated with electron photomicrographs of $M$ ulcerans. The earlier descriptions of the pathology of the disease have been comprehensively reviewed. ${ }^{19}$

In the original paper ${ }^{1}$ MacCallum detailed the clinical and macroscopic appearances of the ulcers with straight or undermined edges, subcutaneous spread producing nodules of induration and eventual ulceration at a distance from the initial lesion (fig 1), and the development in the subcutaneous tissue of "an abundant gelatinous mass like blubbery granulation tissue which could be readily wiped off with gauze". Histologically, necrotic tissue lined the ulcer wall and floor and extensive necrosis of fatty tissue extended beneath the

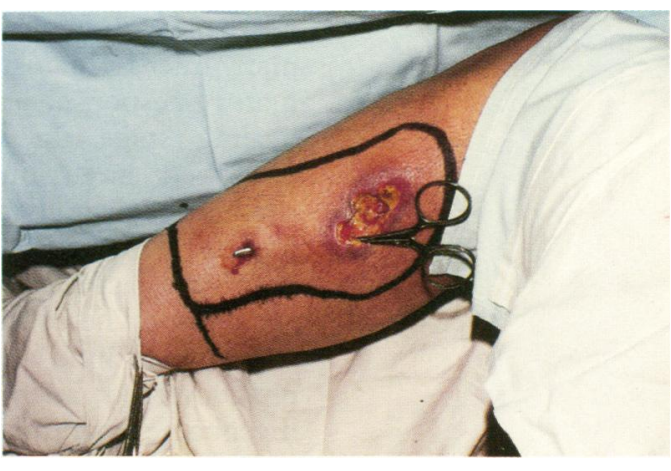

Figure 1 Clinical appearances of Mycobacterium ulcerans infection, on the posterolateral aspect of the left leg in a 64 year old man. The photograph demonstrates the undermining of skin and the development of a satellite lesion associated with extensive necrosis of subcutaneous fat. (Photograph, courtesy of Mr Kendall Francis, FRACS.).

dermis for varied distances. In and around the necrotic areas acid fast bacteria were present in enormous numbers, grouped characteristically in sharply defined oval or rounded masses often as great in diameter as fat cells, as well as in smaller groups and scattered units.

Janssens and his group in the Belgian Congo described the grossly oedematous form of the disease, ${ }^{711}$ also subcutaneous calcification, and noted that the lymph nodes were not affected even in patients with extensive lesions. They used the term "globi" to describe the masses of mycobacteria, a term used to describe the intracellular (within macrophage) collections seen in leprosy, but noted that the acid fast bacilli were constantly extracellular. As well as the acute changes they detailed the long term sequelae occurring after treatment or after spontaneous healing, with formation of granulation tissue at the base and the development of epithelial tissue at the edges of the ulcers. "The scars form very slowly with severe contractions, the ultimate aspect resembles that of a third degree burn, with shortened tendons, ankylosis, muscle atrophy, and lasting lymphoedema."

Dodge, describing the pathology of the Ugandan cases, ${ }^{1213}$ noted necrosis with calcification of the necrotic material to the extent that it may have a gritty consistency. He stated that the necrosis was never caseous, and histologically the degenerate collagen showed a fibrillar pattern with disappearance of fibrocyte nuclei. Necrosis may involve small blood vessels present in the subcutaneous fat. There was a mild inflammatory reaction with occasional giant cell granulomas, in many cases associated 
with large clusters of acid fast bacilli found only focally in the necrotic areas. These form "tangled ribbon-like masses or globular clusters".

Connor and Lunn gave a well illustrated description of 38 Ugandan cases and commented on the difficulty of obtaining unaltered material ${ }^{14}{ }^{15}$; most patients when seen were in late stages when native remedies and secondary bacterial infection had altered the pathological picture. However, Connor was able to detail three histopathological stages: a stage of necrosis with widespread tissue destruction, many micro-organisms and little or no inflammatory response; an organising stage; and a healing stage. In the organising stage he described large numbers of macrophages, plasma cells, and lymphocytes collecting at the margin of the necrotic fat, with proliferating fibroblasts and capillaries growing into the coagulum. The reaction becomes granulomatous as free lipid and necrotic cell debris are phagocytosed; in this healing stage few if any mycobacteria are to be found in sections.

Connor also recorded the vascular changes occurring with the infection, noting eccentric intimal thickening in arteries, which may also be infiltrated by chronic inflammatory cells. Occasionally there is intense infiltration by lymphocytes, oedema of the muscularis, and thrombotic obliteration of the lumen. The changes in the vessels are seen around the perimeter of the necrotic area and were considered to be the result rather than the cause of the necrosis. Connor also described how a biopsy specimen from the proximal edge of an ulcer may show more pronounced healing (granulomatous) changes than one taken from the distal margin, and how this may be reflected clinically in the ulceration progressing slowly down an extremity.

Lytton reported the biopsy material from 54 patients with clinical $M$ ulcerans infection in
New Guinea, ${ }^{16}$ including serial biopsy specimens available from four patients. He suggested that there is a graduation from a "lepromatous" (foamy) type granuloma to the tuberculoid type, analogous to the changes seen in leprosy. ${ }^{20}$

Hayman and McQueen reviewed material from 12 further Bairnsdale patients, including repeated excisions from three patients. ${ }^{17}$ The subcutaneous lesion was recognised as a septate panniculitis. From an initial necrotic process with abundant organisms and minimal inflammation, a changing histological pattern has been observed in the same patients. It is apparent that the entire reactive process resembles the spectrum of leprosy rather than just the granulomas; both types of granuloma are frequently seen in the one patient at the one time and the type of granuloma does not relate directly to the immune state but rather to the necrotic material and the micro-organisms which are present.

Over 45 patients from the Bairnsdale district are now known to have experienced the infection and to have required surgical treatment; the excised tissue has been examined in detail.

Pathogenesis of the infection; the development of a septate panniculitis and progressive ulceration

The disease begins as a dermal lesion with circumscribed necrosis in the deep dermis and adjacent fat, with masses of mycobacteria but little or no inflammatory response. This is followed by epidermal ulceration and development of a granulomatous reaction adjacent to the necrotic area. The disease may remain apparently localised at this anatomical stage of development for months or even years, with clinically apparent healing, scarring, then breakdown and discharge. Microscopic exam-
Figure 2 Histological section of a small ulcer present on thigh for eight months, clinically observed to scab over repeatedly, then break down. Note bland necrosis involving dermis and adjacent fat with a granulomatous response adjacent and deep to the necrotic area. The mycobacteria within the necrotic dermis are present in small clusters; in the deeper area which was once subcutaneous fat they form very much larger masses (arrow) (Ziehl-Neelsen with haematoxylin counterstain).

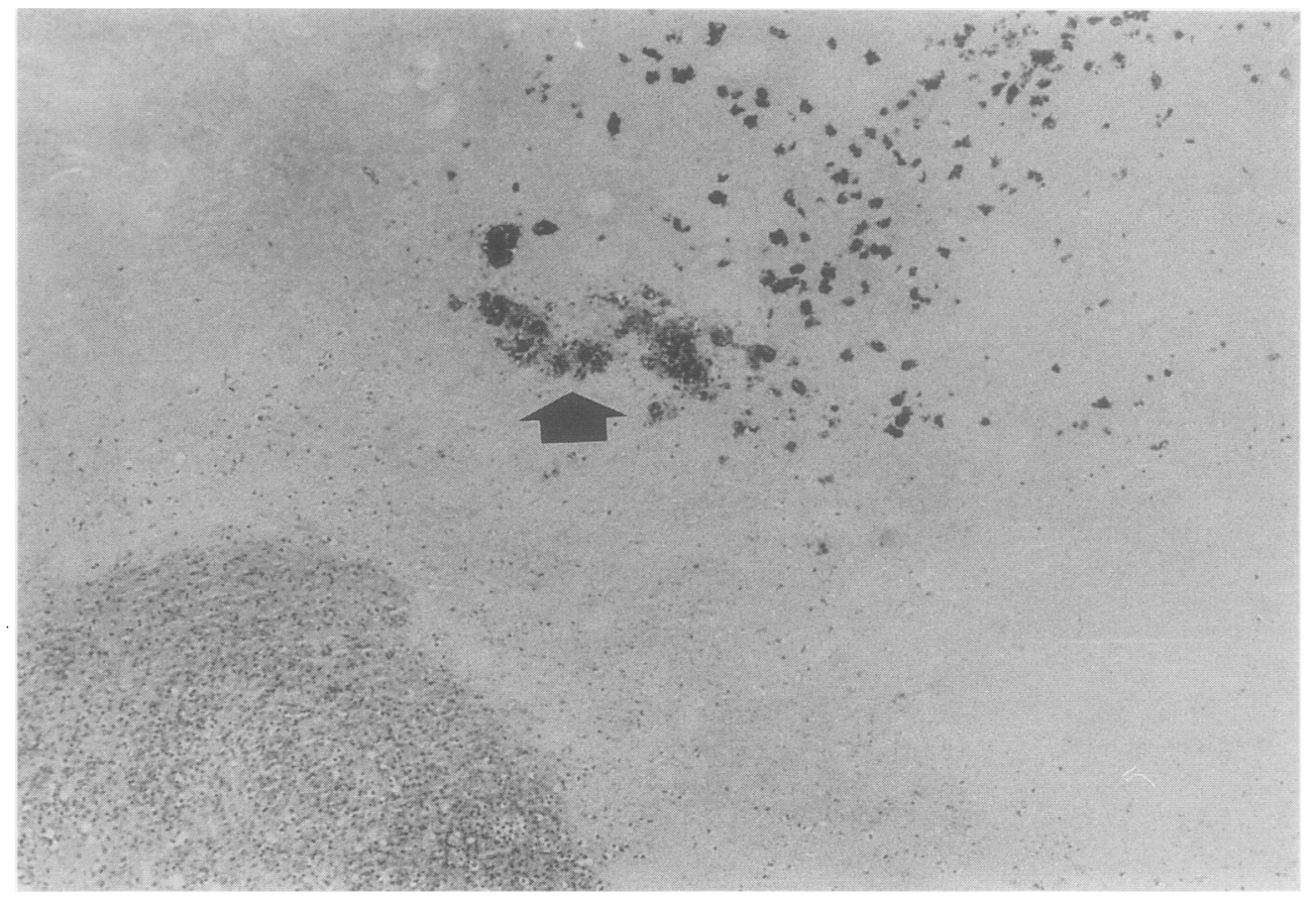


ination in these cases now shows granulation, fibrosis, with epithelial regeneration and hyperplasia occurring beside areas of tissue necrosis and epithelial ulceration. Moderate numbers of acid fast bacilli may still be found, scattered irregularly in the areas of necrosis (fig 2).

In most cases requiring surgery the disease extends deeply to involve the panniculus more extensively, initially with widening of subcutaneous septa due to oedema, increased collagen deposition, and elastosis. Small granulomas and very occasional mycobacteria may be found within these widened septa (fig 3). Similar granulomas also develop superficially in the papillary dermis adjacent to the necrotic area. Initially consisting of small lymphoid collections only, these granulomas later develop into discrete cellular areas with histiocytes and well formed multinuclear giant cells of Langhans' type.

With progressive disease there is gross intimal thickening and occlusion of small arteries within the expanded septa, occurring up to several millimetres distant from the initial infective focus (fig 4). (The mechanism of this change is unknown; it does not seem to be a direct effect of any bacterial toxin production.) This arterial change is followed by subsequent widespread fat necrosis. The fat necrosis represents infarction rather than necrosis occurring as a direct result of mycobacterial invasion and toxin production. Each fat lobule is an end organ; that is, it has a network of capillaries supplied by a single arteriole. The necrosis occurs uniformly throughout the fat lobule and not just in those areas adjacent to the septa where the mycobacteria may be found. From the septa the mycobacteria then pass out into the adjacent necrotic fat where they may multiply into myriads of micro-organisms lying within the lipid lacunae (fig 5).

As well as fat, this secondary necrosis also involves the epidermis and dermis, resulting in

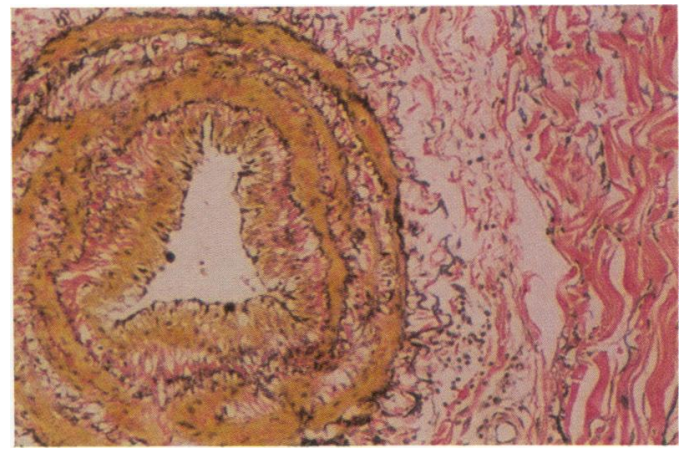

Figure 4 Small artery within an expanded septum in subcutanêous fat, some $6 \mathrm{~mm}$ distant from the area of dermal necrosis. There is intimal thickening and duplication of internal and external elastic laminae (elastic stain with haematoxylin counterstain).

more extensive epidermal ulceration. The skin may, however, be partly spared while the destruction of fat spreads laterally, resulting in the undermining of the epidermis adjacent to the ulcer and often development of satellite ulceration (fig 1).

The changes which follow fat necrosis due to $M$ ulcerans infection are similar to fat necrosis due to other causes in the vicinity of the actual necrotic areas. Thus the necrosis is followed by invasion by macrophages, ingestion of lipid leading to foamy cytoplasmic change, formation of giant cells, and development of granulation tissue that also contains giant cells. This granulomatous reaction appears distinct from the circumscribed granulomas which develop in the dermis and subcutaneous septa at a distance from necrotic areas.

The nature and relevance of the mycobacterial collections

In Ziehl-Neelsen stained sections the mycobacteria within necrotic subcutaneous fat appear as rounded balls or spirals consisting of myriads of organisms, together with smaller
Figure 3 Ulcer excised after six weeks. Widened septum in subcutaneous fat, deep to necrotic area. As well as fibrosis, there is increased elastic tissue in the septa. There are scattered lymphocytes and a small tuberculoid granuloma (arrow).

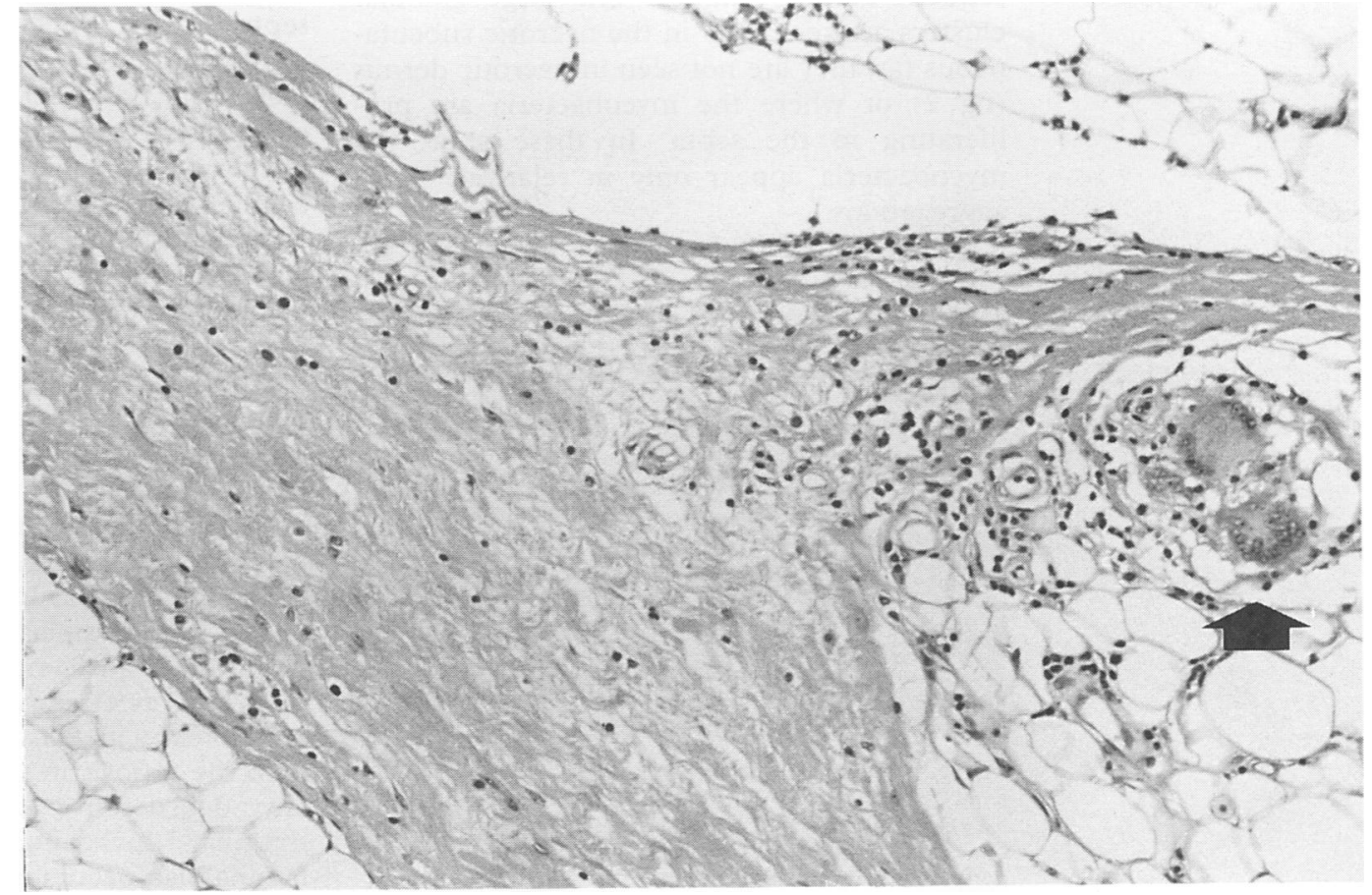


Figure 5 Micro-organisms extending out from necrotic septa (to the left of the photomicrograph) and proliferating in a lipid lacuna within necrotic fat (Ziehl-Neelsen with haematoxylin counterstain).

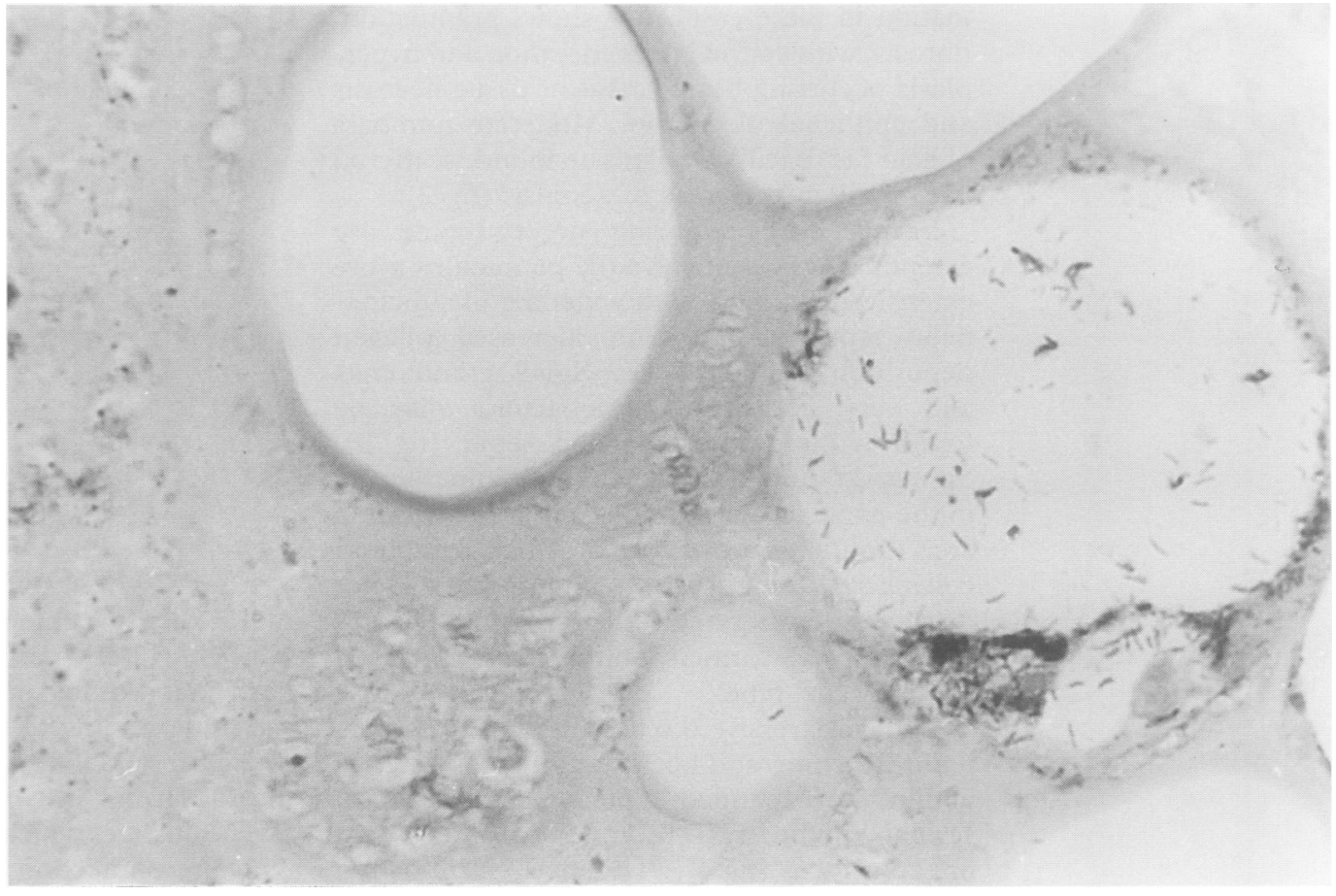

numbers of the bacteria apparently scattered through the necrotic zone. In sections stained with Ziehl-Neelsen and counterstained with haematoxylin this necrotic zone is seen clearly to consist of two components: fatty lacunae or smaller round spaces where the actual lipid material has been dissolved out during processing; and a larger area consisting of proteinacious material, corresponding to the "gelatinous" substance noted clinically and macroscopically. ${ }^{1}$ The mycobacteria are to be found scattered diffusely through some of the large lacunae (fig 5). With progressive absorption of the lipid material the lacunae become smaller and at the same time the bacteria are proliferating, resulting in much smaller lacunae crowded with the organisms, now forming the spherules noted by the original and subsequent authors (fig 6). The large globular clusters are seen only in the necrotic subcutaneous fat; they are not seen in necrotic dermis (fig 2) or where the mycobacteria are proliferating in the septa. In these areas the mycobacteria appear only in relatively small aggregations.

In the globular clusters the organisms are

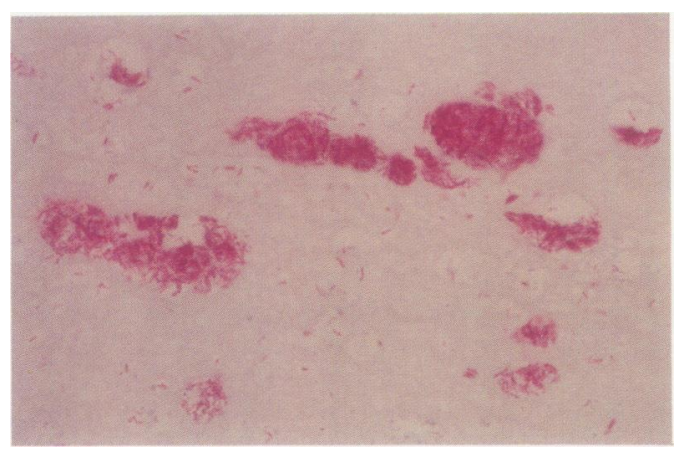

Figure 6 Mycobacteria multiplying in lipid material within necrotic fat forming the large globular clusters which are characteristic of the infection. The name "latebrae" is suggested for these collections (Ziehl-Neelsen with haematoxylin counterstain). extracellular; they survive and multiply in a lipid sanctuary derived from necrotic fat (fig 6 ). It is suggested that the name "globi" should be avoided in describing these collections as the globi of leprosy represent intra-histiocytic mycobacterial collections. Originally the areas are lipid lacunae; the lipid is absorbed and presumably metabolised by the mycobacteria, becoming a mass of micro-organisms rather than a lipid collection. It is proposed that these areas be referred to as latebrae, from the Latin latebra meaning a hide-out or sanctuary.

\section{Connective tissue changes}

TISSUE NECROSIS

In initial lesions there is necrosis of dermal collagen, described by Dodge as "fibrillary" and resulting in an eosinophilic coagulum. This necrosis may extend into the widened septa of the subcutaneous fat as well as involving the dermis. The collagen necrosis may well be described as caseous despite contrary earlier descriptions. Although initially fibrillary, later there is complete loss of all cellular and structural detail resulting in an amorphous eosinophilic coagulum in the affected dermis, histologically indistinguishable from that seen in tuberculous infection (fig 2).

As discussed previously, necrosis of subcutaneous fat occurs as a secondary phenomenon; this is histologically distinct, it has two components in the necrotic areas and it is not caseous. Together with fat and collagen, there is also necrosis and degeneration of elastic fibres. Fragments of elastic fibres may also be seen within the cytoplasm of giant cells in the granulomas adjacent to the necrotic areas.

As well as elastolysis there is also elastosis; an increase in elastic tissue occurs together with the fibrosis in the widened septa prior to any degenerative changes. Duplication or irregular thickening of the internal elastic lamina also occurs in the arteries in the septa 
associated with the intimal proliferation described previously (fig 4).

\section{CALCIFICATION}

Calcification is not prominent in Australian cases but would seem to be an outstanding feature of longstanding and neglected ulcers seen in other countries. Dodge ${ }^{13}$ describes this calcification as "gritty" and calcification is depicted in published radiographs. ${ }^{19}$ Slight calcification, apparent histologically, is noted in many of the Bairnsdale cases in association with fat necrosis and this delicate deposition demonstrates its manner of development. The calcification appears confined to the proteinacious areas between the lipid collections within the necrotic fat, developing around the foci of mycobacterial proliferation.

\section{Conclusion}

Many features of the histopathology of $M$ ulcerans infection are now understood. However, the reasons for susceptibility to infection, the nature of the toxins produced, the mechanism of the initial suppression of the inflammatory response, the production of vascular changes and the changing immune pattern are largely unknown. The Australian distribution of the disease shows that the normal habitat of the mycobacterium is likely to be in tropical rain forest but its isolation from the environment remains unsuccessful despite a search which has now continued for 40 years.
1 MacCallum P, Tolhurst JC, Buckle G, Sissons HA. A new mycobacterial infection in man. I Pathol Bacteriol mycobacterial in

2 Cook A. Mengo Hospital Notes, 1897, Makerere Medical School Library, cited in leading articles. Br Med f 1970; 2:378-9.

3 Radford AJ. Mycobacterium ulcerans: a review, 1: Epidemiology. Papua New Guinea Med $\mathcal{f}$ 1974;17:129-33.

4 Hayman J. Mycobacterium ulcerans: an infection from Jurassic time? Lancet 1984;ii:1015-16.

5 Hayman J. Clinical features of Mycobacterium ulcerans infection. Aust f Dermatol 1985;26:67-73.

6 Uganda Buruli Group. Clinical features and treatment of preulcerative Buruli lesions. (Mycobacterium ulcerans infection): report II. Br Med f 1970;ii:390-3.

7 Janssens PG, Quertinmont MJ, Sieniawski, Gatti F. Necrotic tropical ulcers and mycobacterial causative Necrotic tropical ulcers and mycobacte
agents. Trop Geog Med 1959;2:293-312.

8 Smith JH. Epidemiologic observations on cases of Buruli ulcer seen in a hospital in the lower Congo. Am f Trop Med Hyg 1970;19:657-63.

9 Reid IS. Mycobacterium ulcerans infection; a report of 13 cases at the Port Moresby General Hospital, Papua. Med f A ust 1967;1:427-31.

10 Lunn HF. Mycobacterial lesions in bone. East African Med f 1963;40:1 13-7.

11 Janssens PG. Skin ulcers caused by acid-fast bacilli. In Marshall J, ed. Essays on tropical dermatology Amsterdam Exerpta Medica. 1972:264-95.

12 Dodge OG, Lunn HF. Buruli ulcer: a mycobacterial skin ulcer in a Uganda child. F Trop Med Hyg 1962;65: ulcer in $139-42$.

13 Dodge OG. Mycobacterial skin ulcers in Uganda; histopathological and experimental aspects. $f$ Pathol Bacteriol 1964;88:167-74.

14 Connor DH, Lunn HF. Mycobacterium ulcerans infection (with comments on pathogenesis). Int $\mathcal{f}$ Leprosy 1965;33:698-709.

15 Connor DH, Lunn HF. Buruli ulceration. Arch Pathol 1966;81:183-99.

16 Lytton DG, Lavett J. The pathology of Mycobacterium ulcerans infection in Papua New Guinea. Papua New Guinea Med f 1974;17:150-6.

17 Hayman J, McQueen A. The pathology of Mycobacterium ulcerans infection. Pathology 1985;17:594-600.

18 Burchard GD, Bierther M. Buruli ulcer: clinical pathological study of 23 patients in Lambarené, Gabon. Trop Med Parasitol 1986;37:1-8

19 Connor DH, Meyers WM, Krieg RE. Infection by Mycobacterium ulcerans. In: Binford $\mathrm{CH}$, Connor DH, eds. Pathology of tropical and extraordinary diseases. Washington, PC: Armed Forces Institute of Pathology, DC: Armed

20 Ridley DS, Jopling WH. Classification of leprosy according to immunity. A five-group system. Int $f$ Leprosy 1966;34:255-73. 